\title{
Virtual hands: a comparative study of two text input paradigms for VR
}

\author{
A. Pastor \\ 4 Information Technologies, Multimedia and Telecommunications Department, \\ Universitat Oberta de Catalunya, Barcelona, Spain \\ alvaropastor@uoc.edu

\begin{abstract}
Typing is still the primary input modality for computing systems. Most typical Virtual Reality (VR) setups replace users' capable hands and fingers with cumbersome hand-held controllers (HC). This study examines the hypothesis that finger interaction and realistic representation of users' hands increases typing performance, sense of Presence, and the usability of a typing system for a text transcription task in VR. We developed a hand and finger tracking and visualization system ( $\mathrm{VH}$ ) aimed to help users interact with on-screen keyboards in VR, and compared participants typing performance using $\mathrm{HC}$. We found that the $\mathrm{VH}$ paradigm in VR significantly increased typing performance for inexperienced typists and that $\mathrm{HC}$ users were more prone to commit errors. Further research may delve deeper into the utility of $\mathrm{VH}$ input paradigm for people unable to grasp HCs and for other symbolic communications such as sign language.
\end{abstract}

Keywords: Interaction · Input Interface · Virtual Reality · Keyboard

\section{Introduction}

In recent years, virtual reality (VR) has increased in realism, providing an immersive experience that allows users to deploy their cognitive and physical abilities. However, by using the typical paradigm of head-mounted display (HMD) and hand-held controllers (HC), users lack the critical ability to interact with the system using their hands and fingers. Among all interactions in VR, the keyboard is still the primary text input modality. Its widespread implementation has made users familiar with the standard PC keyboard and proficient in its usage. Although hand and finger interaction remains desirable in order to manually interact with any virtual object, its lack is specially notorious in applications where typing is required. This study examines the hypothesis that finger interaction and virtual representation of users' hands increases typing performance of inexperienced typists. We also examined whether sense of Presence, and the system usability score would significantly correlate with typing performance. We hypothesized that use of $\mathrm{HC}$ paradigm would increase number of typing errors in comparison to text input via hands and fingers interaction. To test these hypotheses, we designed a text transcription experiment in VR following a within-subject design. We developed a hand and finger tracking and 
visualization system aimed to assist users to interact with on-screen keyboards in VR. We compared participants' typing performance using our virtual hands paradigm $(\mathrm{VH})$ to their performance using HC. As objective measures for text input performance we collected correct words per minute (WPM), corrected error rate (CER), and response time until the first correct keystroke (RTK). As subjective measures, we collected in HMD scores for the system's usability and the users' sense of Presence in each of the experimental conditions using previously validated questionnaires.

Previous research on this topic has established that natural interaction using hands and fingers enhances typing proficiency $[1,2]$. However in few studies the sample population is naive of typing skills in computing systems. Aiming to understand this segment of the population, we aimed to test only inexperienced typists, who were not proficient using either on-screen nor physical keyboards, and who had not had previous experience using VR technologies. Furthermore, by developing our $\mathrm{VH}$ input paradigm we aim to contribute a new alternative to input paradigms for VR users that are unable to grasp HCs (e.g. with fewer fingers or muscle atony). This study addressed correlations of objective typing measures to important factors in VR such as system usability and users sense of Presence. By presenting materials both in native language and a foreign language largely unknown to the participants, this study aimed to clarify whether there was an influence of participants' language proficiency in their typing performance. We found that the ability to interact with hands and fingers and visualize them in VR in real time significantly increased typing performance for inexperienced typists.Also a significant increase of CER in the HC users group was found during trials. However, our study was not able to find a significant correlation between input paradigm and users' sense of Presence and system usability ratings.

\section{Related work}

Text input is one of the most common tasks in computing environments including VR systems. Bowman et al.[3] provides a number of possible scenarios of use that demonstrate the potential for alphanumeric input in VR such as the need for entering file names, labeling objects, parameter setting, and communication between users and system. Other scenarios may include working with text in VR, for example VR developers who would not need to frequently switch between the virtual and real world causing increased cybersickness. Researchers and technological developers have proposed a wide variety of different text input solutions for VR, including head/gaze direction, pen and tablet keyboards, virtual keyboards, touchscreen keyboards, speech-to-text and hand/finger gestures $[1,4-8]$. An early comparison of keyboard, pen, and gesture-based techniques showed poor text input rates which were superseded by physical keyboard typing speeds [1]. Research with other text input techniques for VR such as mobile phone keyboard integration and handwritten character recognition confirmed these results [2]. Researchers obtained up to 9.2 WPM by implementing the use 
of the touch screen with hovering capabilities from a smartphone for selecting characters on a virtual keyboard.

Nowadays, most commercially available controllers such as HTC and Oculus support text input on a virtual keyboard by means of pointing. In this paradigm, users point a virtual beam using the $\mathrm{HC}$ onto a character and confirm their selection by pressing a physical button on the HC. Alternatively, a built-in touch pad can be used to move the pointer around. Pointing methods can make use of the QWERTY layout. Due to the fact that many commercially successful VR products use pointing as a general input, text input via pointing has become the most common VR text input method. For instance Playstation VR is shipped with a tracked Playstation Controller, while Google Daydream proposes a HC to interact with the virtual world. Oculus Touch uses HC containing two buttons, a joystick for the thumb and a touchpad for the index finger. HTC vive relies on $\mathrm{HC}$ with a touchpad for the thumb and a trigger button for the index finger [2]. Current text entry methods with $\mathrm{HC}$ employ the pointing paradigm[3] but such method can be tedious and cumbersome. Alternative text input techniques using $\mathrm{HC}$, require a substantial training time for novice typists and are prone to quickly fatigue users [6]. Son et al [9] implemented an alternative for improving $\mathrm{HC}$ performance focusing on the use of thumbs and its results showed that users could achieve a typing speed over 30 WPM. However, they also found that participants required over two hours of practice as they were unable to translate previous typing knowledge and needed to learn a new set of skills. A similar problem was faced by the HoVR typing paradigm for the Microsoft HoloLens [10] which proposed text input in augmented reality applications through a holographic keyboard and a pointer which is controlled using head rotation. Yu et al. [7] studied head-based text entry for VR and combined the concept with gestureword recognition whereby experienced users perform up to 25 WPM. The VR system FaceTouch [11] leverages a touch-sensitive front cover of the HMD and the sense of proprioception to enable text input with up to ten WPM on a virtual keyboard. Bowman et al. [1] presented three interaction techniques designed for virtual environments and employing pinch gloves including a text input and a menu item selection technique. However none of these approaches can profit from the speed and usability known from typing using human fingers, and demand from users the development of a new set of skills.

Image and depth-sensor approaches for hand motion capture have been actively researched since they alleviate the need for instrumentation of the hand - in contrast to marker-based or glove-based approaches. Terajima et al. [12] developed a system which performs 3D motion tracking of human's hand and fingers from images of a single high-frame-rate camera and that recognizes her typing motion in the air and achieved fast real-time recognition of typing motion. Skripcak et al. [13] illustrated a technique applied in interactive visualizations for virtual reality environments employing gestures to increase and decrease numeric input values, while in Hesselmann et al. [14] the authors presented a technique which allows a user to enter numbers on interactive multitouch tabletops using the 10 fingers of both hands. Bailly et al. [15] on the other hand presented 
Finger-Count gestures for multitouch displays, a coherent set of multi-finger and two-handed gestures and present a variation of finger-count technique for gestures.

Early research on image and depth-sensor approaches employed multi-camera setups consisting of multiple calibrated RGB and/or depth cameras to leverage multi-view constraints. However, calibration of these setups has proved tedious especially for non-expert users. Therefore, more recent approaches have focused on hand motion capture from a single RGB or depth camera [16-19]. Commercial products have been developed in this area such as the Leap motion or the Oculus Quest. Leap Motion (LM) is a sensor device that recognizes the user's hands and fingers, while providing interoperability with the head mounted display (HMD) [20]. LM may be attached to the front of the HMD in order to recognize and interpret the 3D locations of the user's hands while presenting the results in real time in the HMD [21-24] attempting to enhance VR experience [25].

Recently, researchers focused on augmenting VR by incorporating a a desktop keyboard and a video stream into the virtual environment aiming to compensate typing difficulties. McGill et al. [8] compared a physical keyboard view, to VR with no keyboard view, VR with partial view and blended view in a study with 16 participants. They found that providing any type of view of the keyboard in VR, partial view or composite, had a positive effect on typing performance. VR text entry rates of $23.6,38.5$ and $36.6 \mathrm{WPM}$ with mean total error rates of $30.86 \%, 9.2 \%$ and $10.41 \%$, respectively, for the three conditions in VR. Using a similar approach Lin et al. [26] used a depth camera to display a point cloud of a user's hands beside a rendered virtual representation of the physical keyboard. Results showed mean text entry rates ranging from 24.3-28.1 WPM and mean total error rates ranging from $20-28 \%$, from 16 participants.

New hardware and software is being developed every year and increasingly, developers have to tackle the implementation of efficient interaction methods for basic operations such as text entry in VR. However, while hand and finger tracking is possible, most commercial platforms still use $\mathrm{HC}$ to interact in virtual environments. Recently the users of Oculus Quest have at their disposal a Virtual Keyboard Overlay as part of the Immersed applications suite, which advertises hand tracking application that would enable users to perform natural typing outof-the-box [27]. Still, it remains an open challenge how to build a VR system that supports natural interaction for accurate and fast text input using both hands and fingers.

\section{Method}

The goal of this study was to evaluate the effect of VH input modality on typing performance of inexperienced typists in VR in comparison to $\mathrm{HC}$ input modality. We developed a VR system that enabled users to interact using their hands and fingers while seeing a virtual representation in the HMD. We collected typing performance data during a transcription task using native and foreign languages 
target phrases. A phrase was presented in the virtual environment and participants were required to correctly type every character as fast they could.

A within subject design was implemented using $\mathrm{VH}$ and $\mathrm{HC}$ experimental conditions. We assessed typing performance via the objective measures of correct words per minute (WPM), corrected error rate (CER), and response time until the first correct keystroke (RTK). Further, we investigated the overall typing experience via subjective measures on the system's usability and the users' sense of Presence by presenting to users corresponding questionnaires in VR, when still under each of the treatments. Comparisons were made on text input performances in the $\mathrm{VH}$ and $\mathrm{HC}$ conditions. We inquired whether subjects in $\mathrm{HC}$ condition were more prone to error, and if this effect was dependent on language proficiency. We investigated correlations between typing performance and the subjective measures of user's experience. An overview of two conditions is shown in Figure 1.

\section{$3.1 \quad$ Subjects}

We asked 50 (34 female) native Spanish speakers aged from 47 to 71 to conduct a simple online test using a desktop PC [28]. This test measured WPM in a 15 phrase text transcription task in English language. Based on their results $(\mathrm{M}=$ 23.74 WPM, SD = 8.45), we invited the 9 worst performing participants each with less than 18 WPM. Seven (three female) unpaid volunteers accepted to take part in this study. Participants were aged from 49 to $71(\mathrm{M}=59.67, \mathrm{SD}=$ 7.65). None of them declared having significant knowledge on English language. All were right handed and wore corrective lenses during the study.

\section{$3.2 \quad$ Apparatus}

The apparatus for this study comprised one individual setup that allowed users to type on an on-screen keyboard while immersed in VR, in the HC condition and in the $\mathrm{VH}$ condition in which a virtual representation of their own hands was provided.

We designed an empty virtual room using Unity 2019.4.10 software. The room featured an on-screen virtual keyboard that enabled users to type. One of the walls served as a phrase display so participants may read the target phrase as they write as fast as possible. The graphic attributes and positions of these elements as well as the type size was constant for each of the conditions. Each of the phrases displayed for each participant were unique and randomly assigned.

Our experiment was running on a Windows $10 \mathrm{PC}$ with an Intel i7-6700, 32GB RAM, and a Nvidia GeForce GTX 1080 graphics card. The target frame rate was set to 90 frames per second (FPS).

For the hand and finger tracking we used a Leap Motion (LM) sensor that supported hand and finger motion tracking as input, attached to a HTC Vive HMD hardware. The summed up calculated latency caused by motion tracking, rendering pipeline, and HMD never exceeded $30 \mathrm{~ms}$ during the study. 


\subsection{Hand-held controller}

We used a pair of the standard HTC Vive HC devices, and we developed a custom visual representation to indicate the position of the pointer in VR. Pressing a key from the on-screen keyboard was only achieved by activating the trigger button over the corresponding spatial location. Only one trigger of each HC at a time was allowed to stroke a key. Haptic feedback was disabled.

\subsection{Virtual hands}

By integrating the LM sensor in our VR setup we were able determine the hands and fingers positions and render them in the HMD accordingly. Our VH experimental condition allowed participants to interact with the virtual on-screen keyboard using their hands and fingers. For this study, the virtual representations of hands were rendered using a custom version of the LM core assets for Unity [29]. Typing was achieved by pointing with any of the fingers towards an onscreen key. The most outstanding finger was computed as the desired pointing coordinates, and horizontal displacement of these as the keystroke event. Only one finger of each hand at a time may stroke a key.

\subsection{On-screen keyboard}

Our on-screen keyboard followed the QWERTY layout and was presented to users with $50 \%$ opacity occupying the left horizontal half and one third of the vertical height of the visible area in the HMD. Keystroke was visualized by changing the color attribute of a pressed key from light blue (RGBA 151, 197, $213,0.5)$ to light red (RGBA 232, 168, 165, 0.5).

\subsection{Phrases}

We used a custom version of the MacKenzie and Soukoreff 500 phrase set [30]. First, aiming to avoid any influence of participants' language proficiency in typing performance [31,32], participants had to transcribe phrases both in their native and a foreign language. Thus an Spanish translation was prepared. Likewise, our experimental design aimed to provide phrase set with constant number of characters for each participant. From the original set and its Spanish translation, we selected 20 phrases each that matched this criterion. The order of presentation of each phrase was randomized for each participant. In sum, every participant was provided with a constant randomized 2400 character set during the experimental trial. The phrases contained no punctuation symbols.

\subsection{Task}

In this study participants had to accomplish a simple text transcription task in VR using an on-screen keyboard in the two experimental conditions: typing 
using $\mathrm{HC}$, and typing using the $\mathrm{VH}$ paradigm. The order of administration of treatments was randomly assigned to each participant.

Once participants were located in the virtual room, phrases were sequentially presented using a distinct light blue (RGBA 151, 197, 213, 0.5), sans-serif font, on one of the walls. As each character was correctly input, the color attribute of the corresponding character changed to black. Only after correct phrase completion, the system provided a new phrase. A total of 40 phrases (20 in English) were provided to each participant in each condition. There was no time limit for task completion.

\subsection{Objective measures}

Words per minute (WPM) The average typing performance is calculated in WPM where one word is defined to be five characters long. Our system logged participants' keystrokes, and the time the participant took to enter the correct character that composed each phrase.

Corrected error rate (CER) One measure as an indicator of the users' typing performance alongside the WPM is the number of errors in the transcribed string. As the system requires correct phrase completion for proceeding to next phrase, corrected error rate was calculated as the number of substitutions users had to perform to change one character into another in relation to the total 1200 required characters for each condition.

Response time until first correct keystroke (RTK) For several applications, the time to react on a specific event using keyboard input is a critical measure of typing performance. Thus, in addition to standard typing metrics of WPM we measured the accuracy and time to first correct keystroke, to evaluate how efficiently participants could interact with the typing system. RTK was measured as the latency in seconds between visual display of a phrase and the first correct keystroke of its first character.

\subsection{Subjective measures}

Presence score (PQ) One of the key characteristics of VR is the ability to create a sense of Presence, the feeling of being or acting in a place, even when one is physically situated in another location [33]. For this study we used an Spanish translation of the 6 item Slater-Usoh-Steed questionnaire (PQ) [34] presented to participants via the HMD in VR, and completed using the on-screen keyboard while under the effects of each treatment. We used a 7 point scale where the highest value corresponded to strong agreement. Previous use of a similar method of presentation indicated that, besides reducing a study's duration and reducing disorientation, completing questionnaires in VR does not change the measured Presence but can increase the consistency of the variance [35]. 
Usability rating (PSSUQ) As typing in VR has been known to be a chal286 lenges among users especially novice [36], we have measured usability with the IBM usability satisfaction survey [37] as it remains a widely used instrument to te usability of software systems. Specifically, we administered via HMD [38] a custom Spanish translation of the 19-item Post-Study System Usability Questionnaire (PSSUQ) which participants completed using each input modality once at the end of each treatment. Our system did not provide error messages while the users interacted, thus item number 9 was excluded. A 7 point scale was used where the highest value matched strong agreement.

\subsection{Procedure}

After welcoming the participants, we asked them to sign the consent form and stand next to the apparatus. We explained the general course of the study and each of the concrete steps to be taken. Afterwards, we proceeded to adjust the HMD to the participant's head and calibrate it to the participant's inter pupil distance for best visual results. Then participants begun the first part of the familiarization run during which the essentials of the main experimental task were explained. A test phrase was showed on the display wall, which allowed participants to spend time trying several locations in the virtual room to achieve the most comfortable reading location. In the second part of this phase the onscreen keyboard is presented in HMD to participants, and tested typing one phrase using each input modality in the same order as they would during the experimental trial. This part had a maximum duration of 60 seconds for each condition after which the on-screen keyboard was removed while the test phrase remained on the wall. We did not include these data in our analysis.

After the familiarization run, participants remained in the virtual room with their HMD on. Before proceeding to the experimental trial, participants confirmed that were able to read the test phrase from their location. Once verification was completed, and without removing the HMD, the test phrase disappeared from the display wall and a 5 seconds countdown was visualized instead to prepare participants for the beginning of the main task. Forty phrases (20 English) were presented sequentially for each condition. Participants were asked to accurately transcribe all of the characters including spaces as fast as possible and were allowed to correct errors. Order of phrases was randomly assigned for each participant. There was no inter treatment delay and no time limit for task completion.

Once the main task was completed, the third phase started. Without removing HMD, participants were presented with the usability and Presence questionnaires directly in the HMD in randomized order and with no inter questionnaire delay. There was no time limit for the completion of this phase. No scores were displayed to participants.

The final step consisted in helping participants to detach the HMDs and then debriefing was conducted. Participants completed all the phases of the study in one session, ranging from 42 to 59 minutes in total duration. 

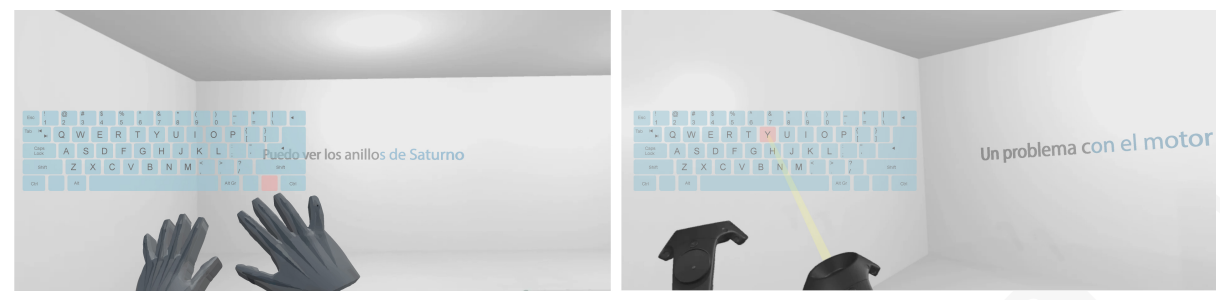

Fig. 1. Two paradigms of text input in VR assessed in this study: Virtual hands (VH) and hand-held controller (HC).

\section{Results}

Seven volunteers took part and completed this pilot study. We assessed the role of input modality in users' performance on the text transcription task. For each condition, participants used our VR system to correctly input 1200 characters in 40 phrases. All significance levels are at $\mathrm{p}=.05$.

We hypothesized that in a VR text input task, VH condition would lead to an increase in typing performance of inexperienced typists measured in WPM and RFK. In addition, we hypothesized that the use of $\mathrm{HC}$ paradigm would increase CER. We also hypothesized that participants with higher ratings in PQ and PSSUQ would significantly outperform others.

When we examined whether VH condition impacted on WPM, a trend was observed for increased typing performance in VH conditions: Using ANOVA we found statistically significant results for $\mathrm{F}=18.913$ and $\mathrm{p}=.005$. We then assessed RTK in respect to the two experimental conditions and found a significant effect of input modality on RFK favoring the VH paradigm at $\mathrm{F}=6.899 \mathrm{p}=.039$.

To test our second hypothesis we measured the CER for each condition and found error rates were overall low (VH mean 1.91\%, HC mean 2.68\%). We evaluated whether users in $\mathrm{HC}$ condition were more prone to commit typing errors. Using ANOVA we found statistically significant increase of error in the HC condition for $\mathrm{F}=10.618 \mathrm{p}=.017$. Further, we investigated if these effects were related to language proficiency in the two experimental conditions. We found no significant difference between language for $\mathrm{VH}$ condition $\mathrm{F}=0.897 \mathrm{p}=.380$. Instead, for $\mathrm{HC}$ condition we found statistically significant increase in CER for English $\mathrm{F}=7.399, \mathrm{p}=.034$.

A Pearson product-moment correlation coefficient was computed to assess the relationship between the mean scores of WPM and the scores on PQ. We found a moderate positive correlation between Presence and input performance for $\mathrm{r}=0.505, \mathrm{p}=.247$ for the $\mathrm{VH}$ condition and a weak correlation between WPM and $\mathrm{PQ}$ score $\mathrm{r}=0.077, \mathrm{p}=.868$ for the $\mathrm{HC}$ condition. We performed a similar analysis to find correlations between PQ scores and CER speculating whether users' sense of Presence affects propensity to commit typing mistakes. While not significant correlations, for $\mathrm{VH}$ we found a moderate positive correlation $\mathrm{r}=0.650$ 
$\mathrm{p}=.114$, and for the $\mathrm{HC}$ condition we found a weak negative correlation between PQ score and CER $\mathrm{r}=-0.107 \mathrm{p}=.819$.

362 We addressed the relation between WPM and usability ratings obtained via PSSUQ. Using Pearson product-moment correlation coefficient for each condition we obtained a non significant negative correlation in $\mathrm{VH} \mathrm{r}=-0.123, \mathrm{p}=.792$, and likewise for $\mathrm{HC}$ condition $\mathrm{r}=-0.048 \mathrm{p}=.918$. To clarify if users typing mishaps may have been evaluated as shortcomings of the VR system, we performed an evaluation of the relation between CER and PSSUQ ratings. A Pearson productmoment correlation coefficient was calculated to find a significant negative correlation $\mathrm{r}=-0.965 \mathrm{pj} .001$ in data from the $\mathrm{VH}$ condition. On a similar analysis performed for the $\mathrm{HC}$ condition, a weak correlation between CER and PSSUQ usability ratings was found $\mathrm{r}=-0.269 \mathrm{p}=.559$.

\section{Discussion}

Our primary aim was to investigate whether the capacity to interact with hands and fingers and visualize them in VR in real time significantly increased typing performance for inexperienced typists.

The results of this study supported our first hypothesis, that participants in the VH condition showed a statistically significant increase in typing performance. Evidence supported our second hypothesis as we analyzed CER in each condition and found a significant increase of typing errors in the HC users group. Nevertheless, our study found only a weak but not significant correlation between input paradigm and users' sense of Presence and system usability ratings. However this correlation did show a trend that may require further examination.

We have shown an experimentally validated setup for studying two text input paradigms in VR. For this study we developed a VH text input paradigm as a solution to widespread problem of text input in VR using HC. Our solution showed increased text input performance while indicating a trend for increased Presence score and usability in VR. There was an altogether positive acceptance of the $\mathrm{VH}$ input paradigm among participants. The impact of enabling users to interact with virtual keyboards using their fingers while visualizing a virtual representation of their hands was demonstrated. As the task relied entirely in character by character transcription skills we found no significant effect on language used during trials. The results of this paper are expected to benefit further research of text input paradigms in VR without requiring cumbersome hardware or learning new skills. Moreover, they may also contribute to research of text input in augmented reality applications.

During this study we evaluated the LM sensor specific and used it to build our apparatus. Positional tracking was accurate, however, future iterations may profit from considering other devices such as the Intel's Real Sense sensor series [39] to achieve more precise tracking and visual accuracy. Future studies may work on optimizing overall system latency for $\mathrm{VH}$ text input paradigm, as enhance accuracy of the $\mathrm{VH}$ visual representation. Interestingly, it was observed 
that all VH paradigm users, which relied on most outstanding finger of each hand as pointing device, used the index and middle finger of the dominant hand and the index finger of the non dominant hand in the text input task, although no previous suggestion was provided. Regarding experimental materials, future research may profit from the use of more challenging phrase sets, e.g. including more difficult punctuation, and the study of native and foreign languages in users transcription abilities [40]. The impact of keyboard shape and the visual attributes of the interactions over text entry performance in VR should also be considered [41].

The findings presented in this pilot study should be interpreted with caution. However its results have pointed towards interesting research directions. Future iterations may delve deeper into participants typing proficiency and learning curve using these paradigms [42-44], comparing novice typists with expert typists. Another approach may inquire further on the utility of such text input paradigm for people unable to grasp HCs (e.g. with fewer fingers or muscle atony) $[45,46]$. Other symbolic forms of communication in VR seem plausible using our VH paradigm. Future studies may address sign language recognition systems which can potentially be derived from the findings in this pilot study, and from our technological framework $[47,48]$.

\section{References}

1. D. Bowman, C. J. Rhoton, and M. Pinho. Text input techniques for immersive virtual environments: An empirical comparison. Proceedings of the Human Factors and Ergonomics Society Annual Meeting, 46:2154 - 2158, 2002.

2. G. González, J. Molina, A. García, D. Martínez, and P. González. Evaluation of text input techniques in immersive virtual environments. In Interacción, 2007.

3. D. Bowman, E. Kruijff, J. LaViola, and I. Poupyrev. 3D User Interfaces: Theory and Practice. Addison Wesley Longman Publishing Co., Inc., USA, 2004.

4. G. Lepouras. Comparing methods for numerical input in immersive virtual environments. Virtual Reality, 22:63-77, 2017.

5. Y. Lee and G. Kim. Vitty: Virtual touch typing interface with added finger buttons. In $H C I, 2017$.

6. J. Grubert, L. Witzani, E. Ofek, M. Pahud, M. Kranz, and P. O. Kristensson. Text entry in immersive head-mounted display-based virtual reality using standard keyboards. 2018 IEEE Conference on Virtual Reality and 3D User Interfaces (VR), pages 159-166, 2018.

7. C. Yu, Y. Gu, Z. Yang, X. Yi, H. Luo, and Y. Shi. Tap, dwell or gesture?: Exploring head-based text entry techniques for hmds. Proceedings of the 2017 CHI Conference on Human Factors in Computing Systems, 2017.

8. M. McGill, R. Murray-Smith, D. Boland, and S. Brewster. A dose of reality: Overcoming usability challenges in vr head-mounted displays. Proceedings of the 33rd Annual ACM Conference on Human Factors in Computing Systems, 2015.

9. J. Son, S. Ahn, S. Kim, and G. Lee. Improving two-thumb touchpad typing in virtual reality. Extended Abstracts of the 2019 CHI Conference on Human Factors in Computing Systems, 2019. 
10. Y. R. Kim and G. J. Kim. Hovr-type: Smartphone as a typing interface in vr using hovering. 2017 IEEE International Conference on Consumer Electronics (ICCE), pages 200-203, 2017.

11. J. Gugenheimer, D. Dobbelstein, C. Winkler, G. Haas, and E. Rukzio. Facetouch: Enabling touch interaction in display fixed uis for mobile virtual reality. Proceedings of the 29th Annual Symposium on User Interface Software and Technology, 2016.

12. K. Terajima, T. Komuro, and M. Ishikawa. Fast finger tracking system for in-air typing interface. In CHI Extended Abstracts, 2009.

13. T. Skripcak, P. Tanuska, and N. Schmeisser. Design and implementation of interactive visualisation configuration using interaction paradigms in virtual reality environment. Int J Soft Comput Eng (IJSCE), 1(5):57-65, 2011.

14. T. Hesselmann, W. Heuten, and S. Boll. Tap2count: numerical input for interactive tabletops. In Proceedings of the ACM interna- tional conference on interactive tabletops and surfaces. ACM, pages 256-257, 2011.

15. G. Bailly, J. Müller, and E. Lecolinet. Design and evaluation of finger-count interaction: Combining multitouch gestures and menus. Int. J. Hum. Comput. Stud., 70:673-689, 2012.

16. F. Mueller, F. Bernard, O. Sotnychenko, D. Mehta, S. Sridhar, D. Casas, and C. Theobalt. Ganerated hands for real-time 3d hand tracking from monocular rgb. 2018 IEEE/CVF Conference on Computer Vision and Pattern Recognition, pages 49-59, 2018.

17. A. Spurr, J. Song, S. Park, and O. Hilliges. Cross-modal deep variational hand pose estimation. 2018 IEEE/CVF Conference on Computer Vision and Pattern Recognition, pages 89-98, 2018.

18. C. Zimmermann and T. Brox. Learning to estimate $3 \mathrm{~d}$ hand pose from single rgb images. 2017 IEEE International Conference on Computer Vision (ICCV), pages 4913-4921, 2017.

19. Yuan S, Garcia-Hernando G, Stenger B, Moon G, Yong Chang J, Mu Lee K, Molchanov P, Kautz J, Honari S, Ge L, and Yuan J. Depth-based 3d hand pose estimation: From current achievements to future goals. 2018 IEEE/CVF Conference on Computer Vision and Pattern Recognition, pages 2636-2645, 2018.

20. T. Ha and W. Woo. Bare hand interface for interaction in the video see-through hmd based wearable ar environment. In ICEC, 2006.

21. H. Mentis, K. O’Hara, G. Gonzalez, A. Sellen, R. Corish, A. Criminisi, R. Trivedi, and $\mathrm{P}$. Theodore. Voice or gesture in the operating room. Proceedings of the 33rd Annual ACM Conference Extended Abstracts on Human Factors in Computing Systems, 2015.

22. G. Park, H. Choi, U. Lee, and S. Chin. Virtual figure model crafting with vr hmd and leap motion. The Imaging Science Journal, 65:358 - 370, 2017.

23. S. Vosinakis and P. Koutsabasis. Evaluation of visual feedback techniques for virtual grasping with bare hands using leap motion and oculus rift. Virtual Reality, 22:47-62, 2017.

24. G. Bruder, F. Steinicke, and W. Stuerzlinger. To touch or not to touch?: comparing $2 \mathrm{~d}$ touch and 3d mid-air interaction on stereoscopic tabletop surfaces. In SUI '13, 2013.

25. J. Han and N. Gold. Lessons learned in exploring the leap motion(tm) sensor for gesture-based instrument design. In NIME, 2014.

26. J.W. Lin, P.H. Han, J.H. Lee, Y.S. Chen, T. Chang, K.W. Chen, and Y. Hung. Visualizing the keyboard in virtual reality for enhancing immersive experience. In SIGGRAPH' '17, 2017. 
27. Immersed. Work faster in vr than in real life, 2020.

28. Aalto University. Aalto typing test, 2019

29. Leap Motion. Leap motion core assets for unity 4.4.0, 2018.

30. I. MacKenzie and R. W. Soukoreff. Phrase sets for evaluating text entry techniques. CHI '03 Extended Abstracts on Human Factors in Computing Systems, 2003.

31. P. Isokoski and T. Linden. Effect of foreign language on text transcription performance: Finns writing english. In NordiCHI '04, page 109-112, 2004.

32. K. Lyons K. and J. Clawson. Analytical evaluation of the impact of phrase set on text entry rates. Proc. Extended Abstracts on Human Factors in Computing Systems (CHI EA). Workshop on Designing ad Evaluating Text Entry Methods., page 109-112, 2012.

33. M.V. Sanchez-Vives and M. Slater. From presence to consciousness through virtual reality. Nature Reviews Neuroscience, 6:332-339, 2005.

34. Usoh M., Catena E., S. Arman, and M. Slater. Using presence questionnaires in reality. Presence: Teleoperators \&6 Virtual Environments, 9:497-503, 2000.

35. V. Schwind, P. Knierim, N. Haas, and N. Henze. Using presence questionnaires in virtual reality. Proceedings of the 2019 CHI Conference on Human Factors in Computing Systems, 2019.

36. A. Sutcliffe and K. Deol. Evaluating the usability of virtual reality user interfaces. Behaviour \& Information Technology, 19:415 - 426, 2000.

37. J. R. Lewis. Ibm computer usability satisfaction questionnaires: Psychometric evaluation and instructions for use. Int. J. Hum. Comput. Interact., 7:57-78, 1995.

38. M. Feick, N. Kleer, A. Tang, and A. Krueger. The virtual reality questionnaire toolkit. Adjunct Publication of the 33rd Annual ACM Symposium on User Interface Software and Technology, 2020.

39. Intel. Real sense depth camera d435, 2019.

40. C. Turner, B. Chaparro, and J. He. Typing on a smartwatch while mobile: A comparison of input methods. Human factors, page 18720819891291, 2020.

41. T. Dube and A. Arif. Impact of key shape and dimension on text entry in virtual reality. Extended Abstracts of the 2020 CHI Conference on Human Factors in Computing Systems, 2020.

42. C. Boletsis and S. Kongsvik. Text input in virtual reality: A preliminary evaluation of the drum-like vr keyboard. Technologies 7, 2019.

43. C. Boletsis and S. Kongsvik. Controller-based text-input techniques for virtual reality: An empirical comparison. International Journal of Virtual Reality, 19:215, 2019.

44. N. Maitlo, Y. Wang, C. Chen, L. Mi, and W. Zhang. Hand-gesture-recognition based text input method for ar/vr wearable devices. ArXiv, abs/1907.12188, 2019.

45. M. Hirota, A. Tsuboi, M. Yokoyama, and M. Yanagisawa. Gesture recognition of air-tapping and its application to character input in vr space. SIGGRAPH Asia 2018 Posters, 2018.

46. Pai Y.S., Dingler T., and K. Kunze. Assessing hands-free interactions for vr using eye gaze and electromyography. Virtual Reality, 23:119-131, 2018.

47. L.D. Haggerty. Adjusting the margins: Harnessing the foundations of american sign language for vr. Frameless, 1-1:22, 2019.

48. D. Economou, M. Gonzalez Russi, I. Doumanis, M. Mentzelopoulos, V. Bouki, and J. Ferguson. Using serious games for learning british sign language combining video, enhanced interactivity, and vr technology. J. Univers. Comput. Sci., 26:9961016, 2020. 